\title{
CatStream: An Integrated Catchment-Stream Water Quality Model
}

\author{
I. Hossain ${ }^{a}$ and M.A. Imteaz \\ ${ }^{a}$ Faculty of Engineering and Industrial Sciences, Swinburne University of Technology, Melbourne, Australia. \\ Email:mimteaz@swin.edu.au
}

\begin{abstract}
Water quality modelling is the primary tool used for catchment and stream water quality investigations. The general architecture of a typical water quality model is the integration of the pollutant processes with the hydrologic and hydraulic approaches. However, due to the lack of specific local information and poor understanding of the limitations of various estimation techniques and underlying physical parameters, modelling approaches are often subjected to producing gross errors. Most of the available water quality models are too simple and/or stochastic in nature. Many of those models perform water quality estimations in isolation, i.e. separate water quality models for catchment and stream analyses. Isolated models may lead to inconsistencies and biased results in the prediction of water quality parameters. On the other hand, there are some integrated water quality models, which are very complex requiring huge physical and chemical data as well as determining many model parameters. This paper presents the development of a simple, integrated and deterministic catchment-stream water quality model to be able to continuously simulate different water quality parameters. The integrated model is comprised of two individual models: the catchment water quality model and the stream water quality model. The catchment water quality model consists of two sub-models: rainfall-runoff model and pollutant processes model. The rainfall-runoff model was developed by considering the time-area method of runoff routing. The model estimates amount of surface runoff generated from a specified catchment for which rainfall data is provided. Water quality parameters were incorporated with the developed rainfall-runoff model, which represents the catchment water quality model. This model estimates the amount of pollutant accumulated on catchment surfaces during the antecedent dry days, and their transportation with surface runoff into waterways and receiving water bodies throughout storm events. Similar to the catchment water quality model, the stream water quality model comprised of two sub-models: the stream flow model and stream pollutant processes model. The stream flow model was developed by considering the Muskingum-Cunge method of stream routing. The stream flow model estimates the rate of water flow into the downstream sections of a particular stream reach. The processes of the same water quality parameters as used in the catchment water quality model were incorporated with the stream flow model which represents the stream water quality model. Final output of the stream water quality model is the concentration of transported pollutants into different downstream sections of a particular stream reach. Finally, the catchment water quality model and the stream water quality model were integrated for the continuous simulation of previously mentioned water quality parameters. For calibration and validation of the model, different published data and reliable source data collected by the Gold Coast City Council (GCCC) were used. Calibration of the catchment water quality model and stream water quality model was performed separately. The calibration results demonstrated the suitability of the developed model as a tool to help with water quality management issues. The major advantage of the developed model is the easy and continuous simulations of water quality parameters associated with surface runoff during any rainfall event. The preparation process of the input data for the model is simple. The capability of the model to simulate surface runoff and pollutant loads from a wide range of rainfall intensities make the integrated model useful in assessing the impact of stormwater pollution flowing into waterways and receiving water bodies and to design effective stormwater treatment measures.
\end{abstract}

Keywords: Integrated Model, water quality model, pollutant processes, calibration 


\section{INTRODUCTION}

The direct determination of the water quality parameters from the experimental results on a wide scale is labour intensive, time consuming and expensive. Consequently, water quality models have great interest to the engineers, scientists who are involved in the field aquatic environment. To address the widespread degradation of aquatic environments, watershed management authorities need appropriate modelling techniques so that they can achieve the legislative requirements of sustainable water resources. The usual practice of water quality modelling is performed through separate models in isolation which may lead to inconsistencies and biased results in the prediction of water quality parameters. Cheah and Ball (2007) found that currently adopted stormwater quality models are unable to reproduce the historical pollutographs accurately and reliably. According to Parker et al. (2002), by recognising the approach of integrated model, earlier forms of system models can be replaced with integrated models. However, the development of an integrated model is a challenging task (Rauch et al. 2002). eWater CRC (2013) developed an integrated water quality model, MUSIC. However, MUSIC is a stochastic model and also do not perform in-stream water quality parameters' degradation and dispersion. On the other hand there are some other integrated models i.e. Infoworks ICM (Innovyze, 2013), HSP-F (USGS, 2013) and SALMON-Q (Wallingford Software, 2013). All these models are complex; require huge input data, which in many occasions become a costly exercise.

This paper presents the development of a simple integrated catchment-stream water quality model, which continuously simulates different water quality parameters. The objective was to develop a model, which requires minimum input data, while having enough accuracy. The integrated model will help watershed management authorities by enabling them to implement economically viable and effective management design, and mitigation strategies to protect aquatic environments from the impact of pollution. This approach places emphasis on all aspects of water quality, including physical and chemical quality, habitat quality and biodiversity (Laenen and Dunnette, 1997).

\section{METHODOLOGY}

The catchment water quality model and the stream water quality model were developed individually. The models of the subsystems were formulated in a consistent way and run in parallel to consider the interactions in both ways or to use information from the downstream system to take control actions in the upstream system. Finally, both of the models were integrated to be able to continuously simulate the mentioned water quality parameters. The output from the catchment water quality model was used as the input for the stream water quality model. In each case, the integration was proposed as a means to overcome the separation and fragmentation.

The catchment water quality model was developed for the continuous simulation of stormwater quantity and quality during the period for which rainfall data are given to the model. The model is comprised of two submodels, i.e. the runoff model and the pollutant model. The runoff model also known as the hydrologic model enables the simulation of catchment hydrological processes via a range of commonly applied rainfall loss models and routing method (time-area method). The runoff model allows the determination of $C L$ using any of the available estimation methods (i.e. constant, linearly varying or exponentially varying), rather than using a fixed rainfall loss value for the duration of rainfall. The pollutant model estimates the amount of pollutant accumulated during the $t_{d}$ and the amount washed-off during surface runoff event. The model also recognises the influence of effective impervious area of catchment surfaces. For the practical application of the model, the minimum allowable value of the $t_{d}$ was assumed to one day ( $24 \mathrm{hrs}$ ). This means that if the $t_{d}$ was less than $24 \mathrm{hrs}$, pollutant build-up on catchment surface was negligible and assumed to be zero. Keeping in mind our objectives, it seemed logical to take the model similar to the widely used model SWMM as the basis for the catchment water quality model. Detailed descriptions of the catchment water quality model are provided in Hossain and Imteaz (2009) and Hossain et al. (2010, 2011). The concept of SWMM was considered because adoption of a widely used equation gives confidence to the practical application of any water quality model (Boorman, 2003).

The stream water quality model was developed to enable the concurrent simulation of two main processes, i.e. stream flow and stream water quality. The stream flow model was developed by considering the wellknown Muskingum-Cunge method of stream routing. Muskingum-Cunge method is less accurate compared to other latest techniques; however to avoid computational complexity and cost of acquiring detailed crosssectional data this method was adopted. The pollutant model was developed by considering physical, chemical and biological changes of stream water quality parameters. Water quality parameters of catchments transfer to a stream through the catchment outlet with surface runoff and are discharged into receiving water bodies. Therefore, a stream water quality model was developed with the same water quality parameters of the catchment water quality model. However, for the stream water quality model, the process of $B O D$ was also 
included. Finally, the catchment water quality model and the stream water quality were integrated for the continuous simulation of the mentioned water quality parameters. The main mathematical processes adopted in developing both the pollutant build-up and wash-off models were described in Hossain et al. (2013); Hossain and Imteaz (2011). A computer program was developed using Visual Basic 6.0 for the formulation of the catchment and stream water quality models and their integration.

\section{DEVELOPED MODEL}

Figure 1 shows the basic window of the developed model, through which selections of the models will be performed. Catchment water quality model was developed by considering two submodels: catchment hydrology and catchment water quality. Figure 2 presents the window for catchment hydrologic model. It requires rainfall data, rainfall loss parameters and catchment characteristics data. The model allows user to take rainfall data from .txt file. By pressing the "Read Rainfall Data" button at top left corner the window shown in Figure 2 will allow the users to select rainfall data path from the computer directory.

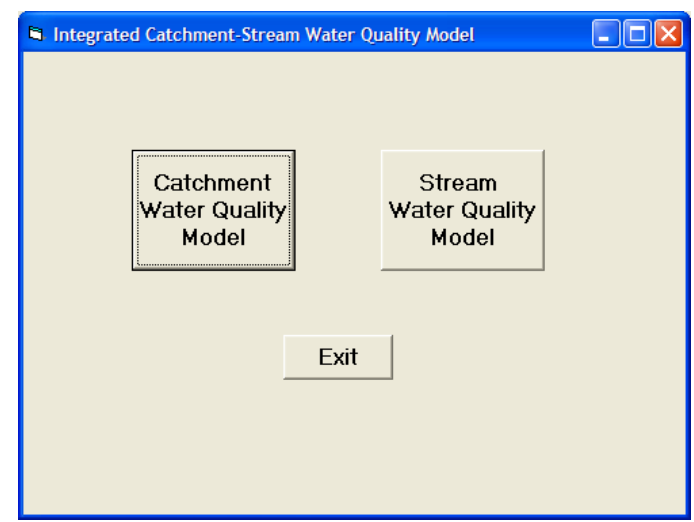

Figure 1. Basic window for the individual model selection

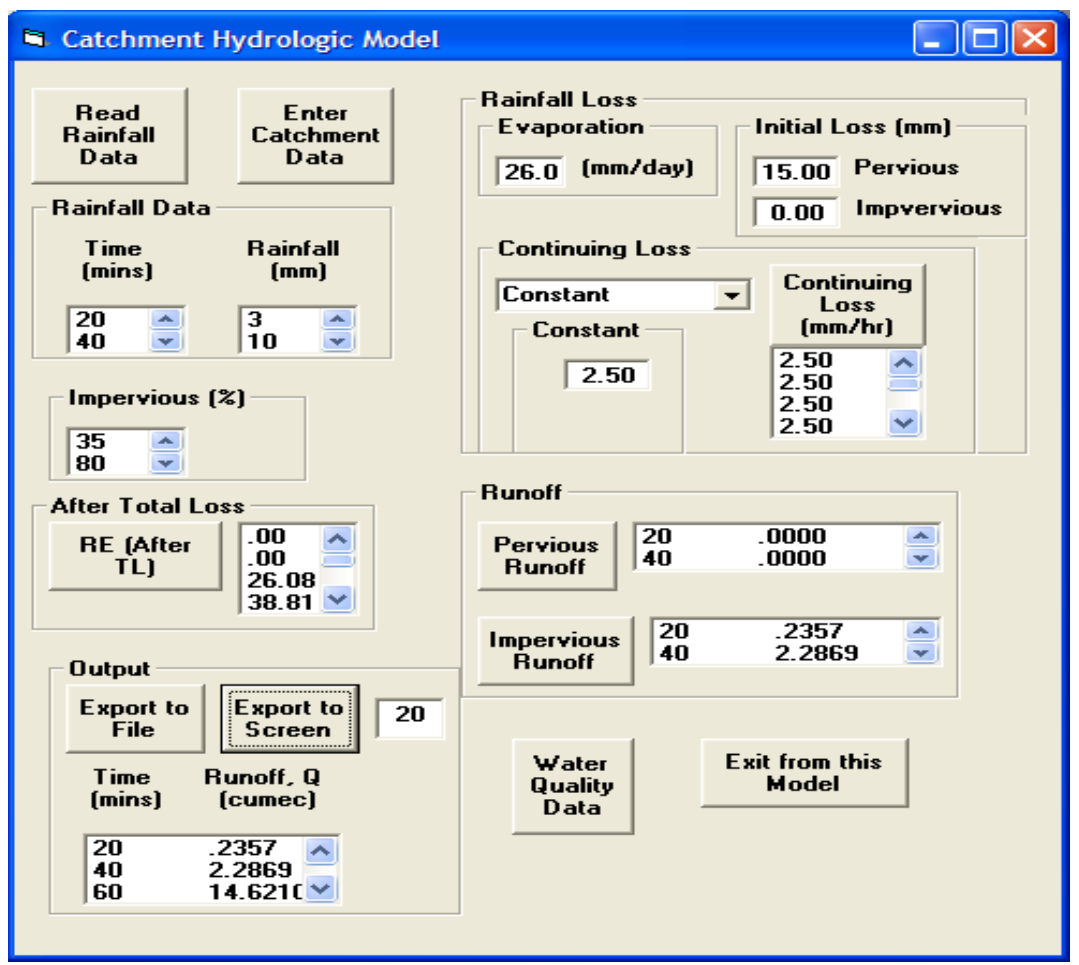

Figure 2. Structure of the catchment hydrologic model

For the generation of surface runoff and pollutant concentrations, like other models the developed model requires catchment characteristics data. By pressing "Enter Catchment Data" button, the hydrologic model allows the users to select catchment characteristics data. Figure 3 shows the window to insert and select the 
catchment characteristics data. There are two different options to provide the sub-catchment and corresponding impervious fraction data. If sub-catchment area and their impervious fractions are known, the user can provide by .txt file. Otherwise, the model can sub-divide the catchment into a suitable number of sub-catchments indicated by the users according to the time of concentration. Also the users have got option to select any of the methods (Bransby Williams, Pilgrim and McDonald and Flavelt equations) to calculate time of concentration.

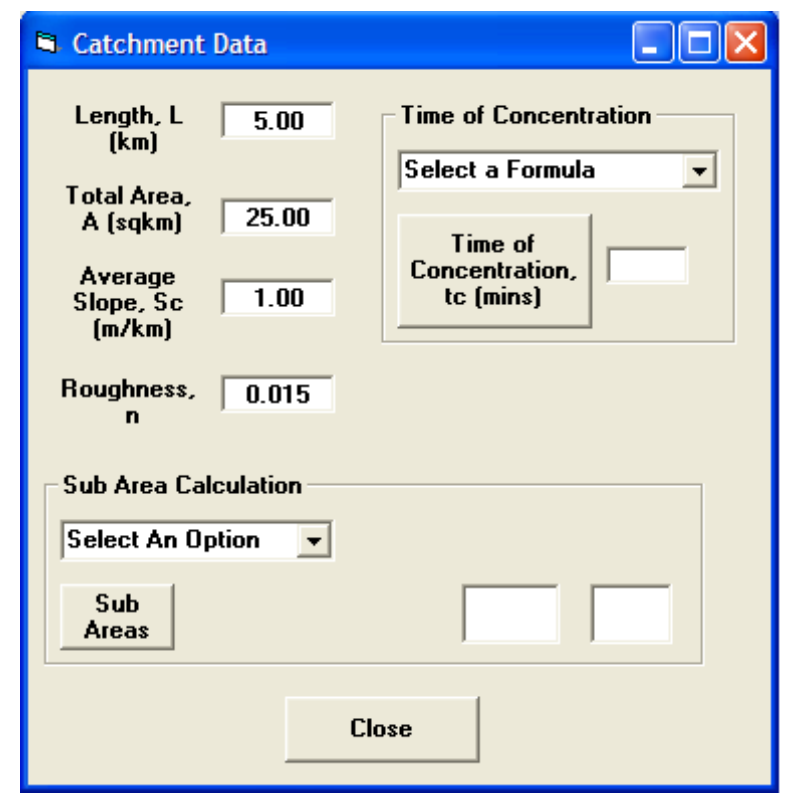

Figure 3. Catchment data window

Evaporation data should be inserted as mm/day. The model allows user to used separate initial loss for impervious and pervious surfaces as constant values. The continuing loss model can be selected from any of the constant, linearly varying or exponentially varying loss models. Once all the input parameters for the hydrologic model are inserted, the model produces surface runoff which washes away the pollutants of the catchment to the downstream environment. Pressing the "Export to File" or "Export to Screen" button shown in Figure 2 will initiate all the calculations related to surface runoff generation.

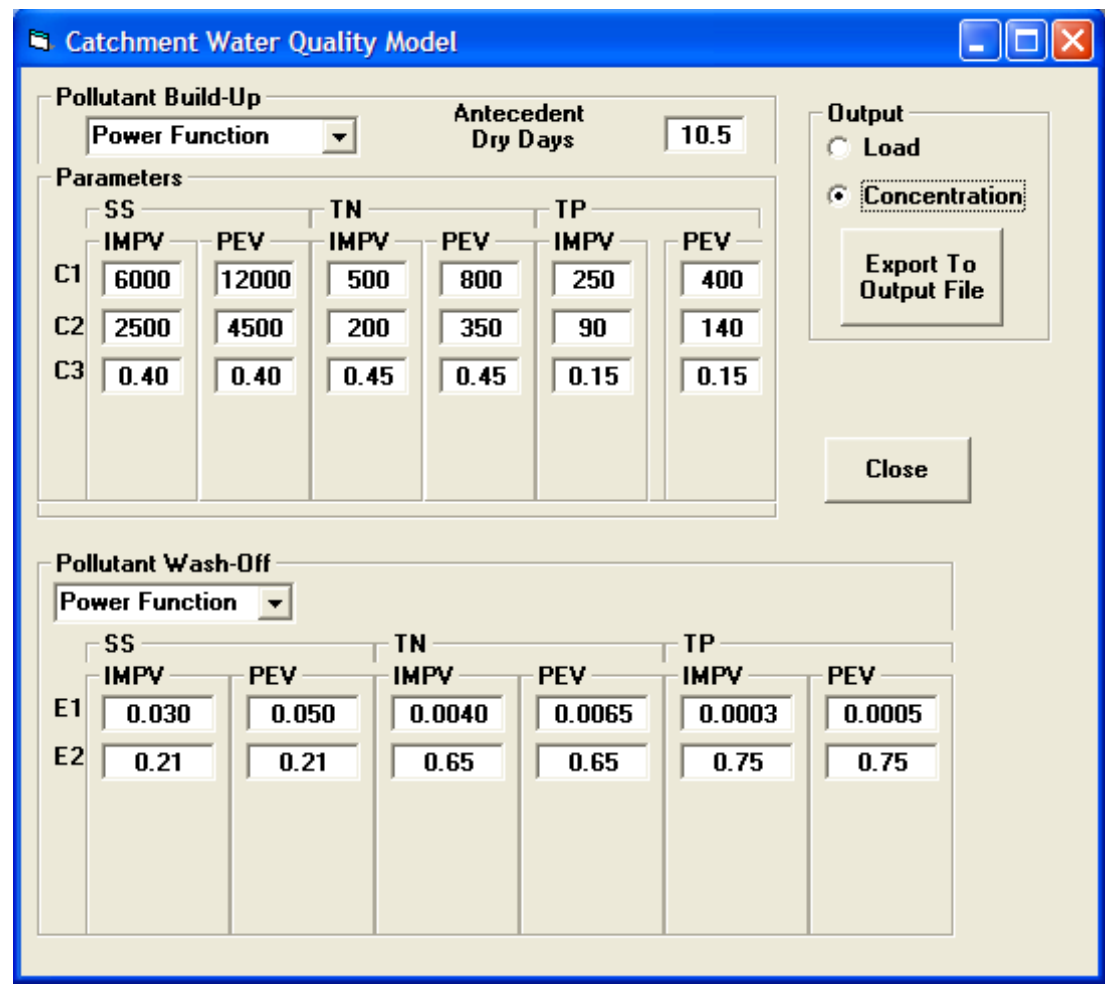


Figure 4. Catchment water quality data window

It is well established that during the antecedent dry days pollutants accumulated on the catchment surfaces and washed off during the rainy days. Therefore, the model uses separate pollutant build-up and wash-off functions. Figure 4 presents the window for the input parameters for the pollutant build-up and wash-off models. Antecedent dry days for the build-up functions can be inserted to the model directly from the historical rainfall data. Intermediate dry days are calculated by the model itself. The catchment water quality model allows user to select any of the three build-up models (power function, exponential function and saturation function) and wash-off (power function, rating curve and saturation function) models. Pressing the button "Export To Output File" shown in Figure 4 will initiate pollutants concentration at catchment outlet. The concentration or pollutant loads from the catchment water quality model can be exported in a .txt file.

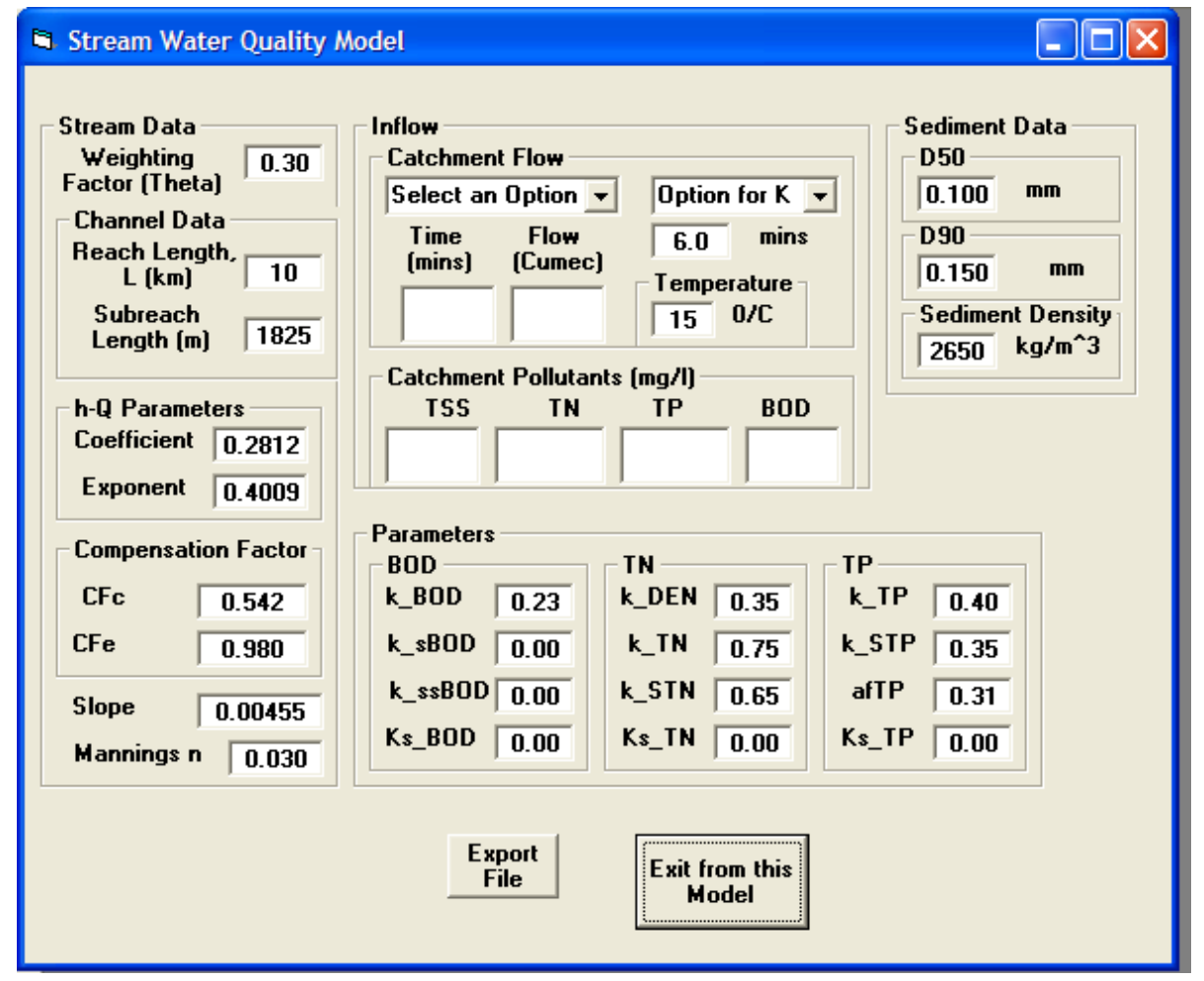

Figure 5. Stream water quality model window

The surface runoff and pollutant loads generated from catchment surfaces will transferred to downstream environment through stream. Therefore, a stream water quality model was developed and integrated with the catchment water quality model. Figure 5 presents the window for stream water quality model. Input parameters for the stream water quality model can be taken directly from the catchment water quality model or can be inserted from a .txt file. Final stream flow and pollutant concentration at the downstream section can be exported in a .txt file by pressing the button "Export File" shown in Figure 5.

\section{RESULTS AND DISCUSSION}

Applications of the developed integrated model were demonstrated for several catchments in Gold Coast, Australia (Imteaz et al., 2014 and Hossain et al., 2012). In this paper, the application of the model was performed for the prediction of suspended sediment (SS) processes, including the transportation and the deposition of sediment for a particular stream reach. The model was applied for the both transportation and deposition processes of $S S$ along the reach section of Saltwater Creek located in the Gold Coast region of Australia. The creek was characterised by a variable hydraulic regime. The length of the studied reach was approximately $1,825 \mathrm{~m}$. For this length the two catchments were contributing surface runoff and pollutant loads. The area of the catchments were 1,025 ha and 245 ha having imperviousness $33.5 \%$ and 53\% respectively. The calibration results of the simulation are shown in Figure 6.

Figure 6 presents the observed and simulated transportation rate of SS for Saltwater Creek during the two consecutive rainfall events. From the figure, it is clear that during the beginning and the end of the runoff simulation, there was increased $S S$ transportation. This was due to the higher concentration of incoming sediment from the contributed catchments of the creek. When there was an increased amount of incoming $S S$ 
in the creek, there was more transportation. However, during this time the stream flow was low. Hence, there should have more $S S$ deposition instead of transportation. As the stream flow was low, the volume of water decreased. Therefore, the concentrations of $S S$ per unit volume of water increased with more incoming $S S$.

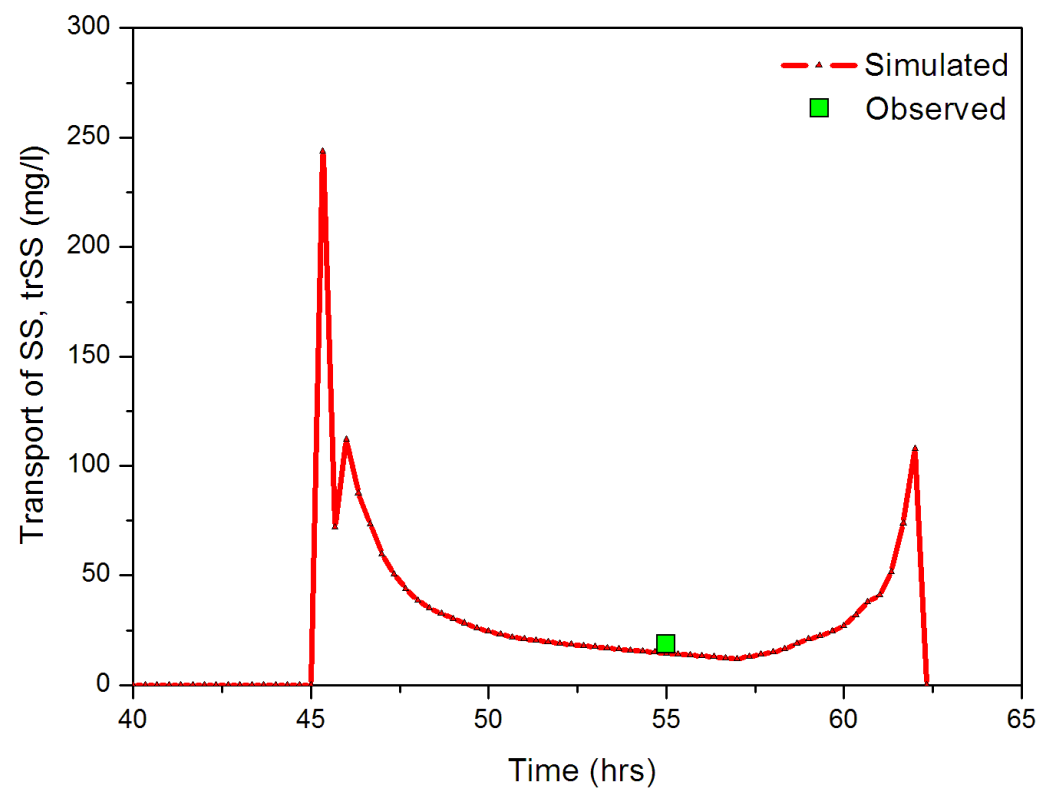

Figure 6. Simulation of $S S$ transport for the Saltwater Creek

On the other hand, there should be more $S S$ deposition with the increased incoming sediment concentration. However, when the transport capacity of the streamflow increases the deposition rate decreases. For the creek, the transport capacity of the flow was strong enough to carry all $S S$ to the downstream at that time. Hence, the transportable $S S$ concentration was higher during the beginning and the end of the simulation even with the higher incoming sediment. However, the observed concentration is very much closer to the simulated results indicating the good performance of the model simulations shown in Figure 6 . It is to be noted that due to the lack of observed data, model performance was shown only for one observation/measurement. To be able to claim model's capability, comparison with more observed data is needed.

\section{CONCLUSIONS}

The modelling technique plays an important role for the development of appropriate watershed management strategies and to support decision making processes. Earlier developed integrated models are either stochastic or too complex requiring too many input data. This paper presents development of an easy, less dataintensive integrated catchment-stream water quality model for the continuous simulation of different quality parameters. The equations used in the model are physical process based; however include several empirical terms and coefficients, which must be determined by the calibration procedure. The major advantage of the model is fewer requirements of input data and the continuous simulation of water quality parameters through an integrated modelling approach. Accurate hydraulic simulations of water quality parameters require detailed cross-sectional data of the creek/river sections, which in most cases is a costly exercise. Through implementation of several techniques, the developed model avoids the need for detailed cross-sectional data while maintaining sufficient accuracy. The requirement of detailed cross-sectional data was replaced through introduction of a new creek/river shape factor.

Comparing the modelling results with the commercially used models, it was found that this model produces similar results. It can therefore be concluded that the proposed technique is promising and may be beneficial to overcome the burden of using individual models. The model can be used to extend water quality surveillance and to predict the future water quality/quantity conditions. The disadvantage of the model is the need for the accurate field measurement data to calibrate the model parameters; nonetheless this requirement is unique for any reliable hydrologic/hydraulic model. There is also a loss of accuracy, because of simplified representation of the physics of the relevant modelling processes. Another disadvantage is that the model does not consider the cohesive properties of $S S$ particles, such as flocculation, consolidation and fluidisation. These processes were ignored in order to make the model easy and less-complex. Finally, it is recommended to apply and compare the model results with a longer period data having several measurements. 
Hossain and Imteaz, CatStream: An integrated Catchment-Stream Water Quality Model

\section{REFERENCES}

Boorman, D.B. (2003) LOIS in-stream water quality modelling. Part 1. Catchments and methods. The Science of the Total Environment, 314-316, 379-395.

Cheah, C.H. and Ball, J.E. (2007) An alternative approach to modelling stormwater runoff from small urban catchment. International Journal of Water, 3(4), 322-333.

eWater CRC (2013) www.ewater.com.au/products/ewater-toolkit/urban-tools/music/ accessed on 27 August, 2013.

Hossain, I., Imteaz, M.A., Arulrajah, A. and Hossain, M.I. (2013) Continuous Simulation of Suspended Sediment through a Stream Section. International Journal of Water, 7(3), 206-222.

Hossain, I., Imteaz, M.A. and Hossain, M.I. (2012) Application of a Catchment Water Quality Model for an East-Australian Catchment. International Journal of Global Environmental Issues, 12, 242-255.

Hossain, I. and Imteaz, M.A. (2009) Development of a Deterministic Catchment Water Quality Model, 32nd Hydrology and Water Resources Symposium, Newcastle, November.

Hossain, I. and Imteaz, M.A. (2011) Continuous Simulation of Suspended Sediment Transportation along a Stream Section, 34th IAHR World Congress, Brisbane, June.

Hossain, I., Imteaz, M. A., and Gato, T. S., and Shanableh, A. (2010) Development of a Catchment Water Quality Model for Continuous Simulations of Pollutants Build-up and Wash-off, International Journal of Civil and Environmental Engineering, World Academy of Science, Engineering and Technology, 2, 210217.

Hossain, I., Imteaz, M.A., Hossain, M.I. (2011) Application of build-up and wash-off models for an EastAustralian catchment, International Journal of Civil \& Environmental Engineering, World Academy of Science, Engineering and Technology, 75 (162), 942-947.

Imteaz, M.A., Hossain, I. and Hossain, M.I. (2014) Estimation of Build-up and Wash-off Models Parameters for an East-Australian Catchment, International Journal of Water, 8(1), 48-62.

Innovyze (2013) InfoWorks ICM, http://www.innovyze.com/products/infoworks_icm/, accessed on 27 August, 2013.

Laenen, A. and Dunnette, D.A. (1997) River quality: Dynamics and restoration. CRC Lewis, Boca Raton, New York.

Parker, P., Letcher, R., Jakeman, A., Beck, M.B., Harris, G. and Argent, R.M. (2002) Progress in integrated assessment and modelling. Environmental Modelling and Softwater, 17(3), 209-217.

Rauch, W., Bertrand-Krajewski, J.L., Krebs, P., Mark, O., Schilling, W., Schutze, M. and Vanrolleghem, P.A. (2002) Deterministic modelling of integrated urban drainage system. Water Science and Technology, 45(3), 81-94.

USGS (2013) Hydrological Simulation Program - Fortran (HSP-F), http://water.usgs.gov/cgibin/man_wrdapp?hspf, accessed on 27 August, 2013. 